\title{
El incendio del 17 de abril de 2003 en Chillogallo (Quito)
}

L'incendie du 17 avril 2003 à Chillogallo (Quito)

The April 17, 2003 Chillogallo fire (Quito)

\section{Jairo Estacio}

\section{(2) OpenEdition}

Journals

\section{Edición electrónica}

URL: http://journals.openedition.org/bifea/2319

DOI: 10.4000/bifea.2319

ISSN: 2076-5827

\section{Editor}

Institut Français d'Études Andines

\section{Edición impresa}

Fecha de publicación: 1 diciembre 2009

Paginación: 527-543

ISSN: 0303-7495

Referencia electrónica

Jairo Estacio, «El incendio del 17 de abril de 2003 en Chillogallo (Quito) », Bulletin de l'Institut français d'études andines [En línea], 38 (3) | 2009, Publicado el 01 junio 2010, consultado el 18 noviembre 2020. URL : http://journals.openedition.org/bifea/2319; DOI : https://doi.org/10.4000/bifea.2319

\section{(c) $(9) \odot$}

Les contenus du Bulletin de l'Institut français d'études andines sont mis à disposition selon les termes de la licence Creative Commons Attribution - Pas d'Utilisation Commerciale - Pas de Modification 4.0 International. 


\title{
El incendio del 17 de abril de 2003 en Chillogallo (Quito)
}

\author{
Jairo Estacio*
}

\section{Resumen}

Importantes infraestructuras relacionadas con el transporte y almacenamiento de combustible se encuentran emplazadas en el Distrito Metropolitano de Quito (DMQ), obedeciendo a un marco de políticas de desarrollo industrial emprendidas en el Ecuador desde la década de 1970. Sin embargo, varias de estas infraestructuras se vieron, con el paso del tiempo, encerradas en el tejido urbano, cada vez más denso, lo que puso en evidencia un peligro potencial para la población. Eso fue lo que pasó con el incendio del año 2003, originado por uno de los poliductos de gasolina que se rompió y atravesaba dos barrios pobres en proceso de consolidación urbana: El Tránsito y El Girón de Chillogallo, ubicados al sur de Quito. Este accidente ocasionó graves daños en la población y en lo material, llegando a ser considerado como uno de los mayores eventos ocurridos en Quito relacionados con el transporte de combustible. El accidente sacó a relucir debilidades en las políticas de prevención y manejo de emergencia. También dejó varias lecciones y experiencias en el campo del conocimiento y gestión del riesgo tecnológico muy poco conocido y priorizado en el DMQ.

Palabras clave: incendio, combustible, poliducto, riesgo tecnológico, peligro, vulnerabilidad, planificación preventiva, gestión de crisis, Quito

\section{L'incendie du 17 avril 2003 à Chillogallo (Quito)}

\section{Résumé}

De nombreuses infrastructures de transport et de stockage de combustibles se concentrent dans le District Métropolitain de Quito (DMQ) en raison des politiques de développement industriel engagées en Équateur depuis les années 1970. Cependant, plusieurs de ces infrastructures ont été progressivement

* Laboratorio Edytem, Département de Géographie, Université de Savoie, Chambéry, France. E-mail: jairo.estacio@univ-savoie.fr. 
englobées dans le tissu urbain, toujours plus dense, ce qui a mis en évidence un danger potentiel pour la population. C'est ce qui s'est produit lors de l'incendie de 2003 suite à la rupture d'une conduite transportant de l'essence qui traverse deux quartiers pauvres en voie de consolidation, El Tránsito et Girón de Chillogallo, situés au sud de Quito. Cet accident fut à l'origine d'importants dommages sur les plans humain et matériel au point d'être considéré comme l'un des évènements les plus graves, liés au transport de combustibles, survenus à Quito. L'accident a mis en évidence des faiblesses tant dans le domaine de la prévention que dans celui de la gestion des crises. Il a aussi apporté une expérience et des enseignements, en termes de connaissances et de gestion, dans le domaine du risque technologique très peu connu et non considéré comme prioritaire dans le DMQ.

Mots clés : incendie, combustible, pipe-line, risque technologique, aléa, vulnérabilité, planification préventive, gestion de crise, Quito

\title{
The April 17, 2003 Chillogallo fire (Quito)
}

\begin{abstract}
Much of the transport and fuel storage infrastructure is concentrated in the Quito Metropolitan District (DMQ). This situation reflects industrial development policies that were implemented in Ecuador since the 1970s. Many of these facilities were gradually surrounded by an increasingly dense urbanization. For this reason, a potential danger to the population has been created and is highlighted in this essay. This potencial threat was materialized with the fire that occurred in 2003, following the breaking of a pipeline carrying gasoline. The pipeline crosses two poor neighborhoods - El Tránsito and Girón de Chillogallo-, located south of Quito. This accident was the cause of significant human and material damage. It was considered as one of the most serious accidents, involved in transporting fuel, that has occurred in Quito. The event has highlighted weaknesses in both the field of prevention and in crisis management. It also provided experience and lessons in terms of knowledge and management in the field of technological risk, subjects which were poorly known and had not been prioritized in the DMQ.
\end{abstract}

Key words: fire, fuel, pipeline, technological risk, hazard, vulnerability, preventive planning, crisis management, Quito

\section{LOS ACONTECIMIENTOS Y SU CONTEXTO}

El accidente en el sector de Chillogallo es consecuencia del riesgo generado por ciertos elementos tecnológicos vinculados con la industria hidrocarburífera en el área urbana. En efecto, el Distrito Metropolitano de Quito, dada sus características de ciudad capital y su rol estratégico para el abastecimiento de combustibles (no solo para el mismo distrito sino para el sur de la región Sierra del país), es un sitio de emplazamiento de infraestructura relacionada con el almacenamiento y transporte, a gran escala, de derivados de petróleo (Estacio, 2004)1.

1 Datos adicionales del sistema de abastecimiento de combustibles son analizados en este volumen en el artículo de J. Estacio «Construcción y transformación del riesgo tecnológicos: la terminal de combustibles El Beaterio-Quito», pp. 683-707. 
Debido a esta particularidad territorial varios han sido los accidentes registrados en las instalaciones hidrocarburíferas, en especial en poliductos y camiones cisterna de transporte de derivados de petróleo. Desde 1970 hasta 2006 se han registrado 118 accidentes tecnológicos en el DMQ, de los cuales al menos 45 se relacionan con hidrocarburos2. Estos representan el $37 \%$ de los accidentes y son más frecuentes que los relacionados a industrias, obras civiles o técnicas3. Dentro de este grupo, el accidente de Chillogallo es el más representativo por el incendio de gran magnitud relacionado con el transporte de combustible a través de poliductos en zonas urbanas.

El incidente ocurrió alrededor de las 3:00 de la madrugada del 17 de abril de 2003, en el sector de El Mirador de Chillogallo, entre los barrios El Tránsito y El Girón de Chillogallo en la parte suroccidental de la ciudad (fig. 1). Un gran incendio seguido de 3 explosiones fueron registrados a la altura de la quebrada El Tránsito (también Ilamada San Vicente), colindante a los dos barrios. Según fuentes oficiales de la vicepresidencia de PETROCUADOR4, el incendio y la explosión fueron provocados por el derrame de cerca de 27 mil barriles de gasolina súper, debido a una fuga del poliducto Quito-Santo Domingo-Esmeraldas. La gasolina siguió, pendiente abajo, el curso del riachuelo de la quebrada, concentrándose finalmente en una zona más plana, encañonada entre las calles Luis Duque y Carlos Freire. Inmediatamente la concentración de gasolina emitió fuertes gases que fueron percibidos a una distancia de 2 kilómetros de la zona. Según un informe oficial emitido por el Cuerpo de Bomberos de Quito:

«(...) se acumularon los gases emanados por el combustible, los mismos que al contacto con un punto de ignición produjeron un fogonazo $^{5}$ y una explosión de los gases en el ambiente. La onda explosiva destruyó una vivienda y muchas otras fueron afectadas. El fuego se propagó por toda el área donde se encontraban los gases del combustible derramado,

2 La construcción del registro de accidentes se basó en la información encontrada en varias instituciones tales como: ministerio del Ambiente, Fundación Natura, PETROECUADOR, el CRED (Centro de Investigación sobre Epidemiología de Desastres, Bruselas-Bélgica), la Red (base DesInventar), y otros organismos operativos entre los que están: el SIAT de la Policía Nacional (Sistema de Información de Accidentes de Tránsito), el Cuerpo de Bomberos de Quito y la Dirección de Planificación Territorial del Municipio Del Distrito Metropolitano de Quito (datos del Sistema de Información Metropolitana). El registro se reforzó con el análisis de prensa de los periódicos El Comercio, El Hoy y Últimas Noticias publicados entre 1970 y 2006.

3 Los accidentes en obras civiles o técnicas están relaccionados con el sector de la construcción (viviendas, equipamientos para la ciudad).

4 PETROECUADOR es la Empresa Estatal Petrolera que maneja la exploración, explotación, transporte, industrialización y comercialización de hidrocarburos. Cuenta con filiales como PETROPRODUCCIÓN que realiza la exploración de hidrocarburos; PETROINDUSTRIAL a cargo de la refinación y producción de productos derivados de petróleo; PETROCOMERCIAL encargada básicamente del transporte, almacenamiento y comercialización de derivados de petróleo y SOTE a cargo del transporte de crudos pesados por oleoducto.

5 El fogonazo hace referencia a una llamarada instantánea que algunas materias inflamables (en este caso gasolina) producen en la combustión. Este tipo de llamarada puede asociarse a un tipo de incendio superficial de gran expansión. 


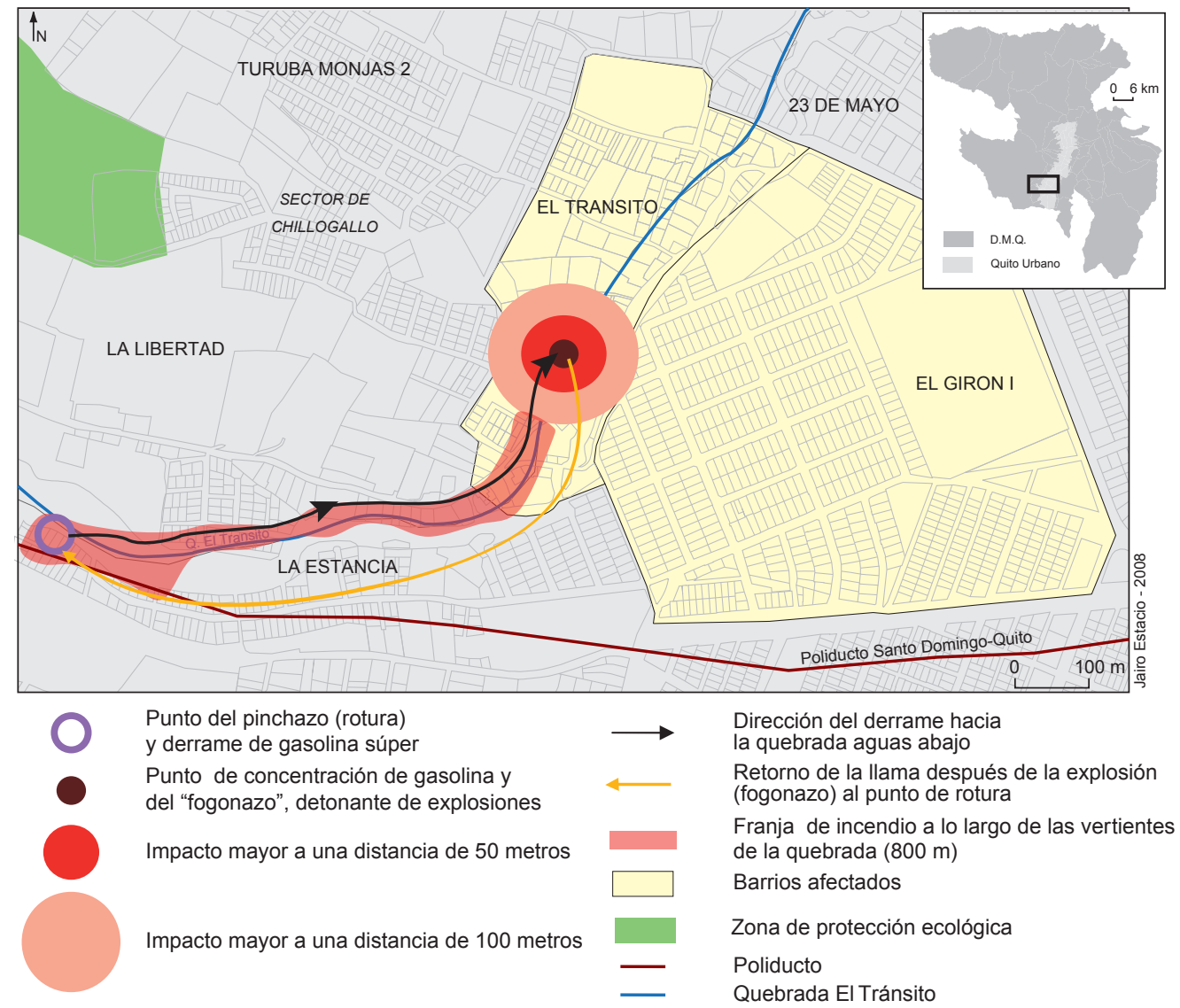

Fuentes: Informes Oficiales Cuerpo de Bomberos Quito y PETROCOMERCIAL, Recortes de prensa El Comercio, Universo, Ultimas Noticias, El Hoy, Telégrafo, Abril 2003, SUIM-DMPT-MDMQ

Figura 1- Localización del incendio de Chillogallo (abril del 2003)

produciéndose un incendio de grandes proporciones a lo largo de la quebrada y en especial en el punto de la ruptura de la tubería que transportaba el combustible».

Según testigos oculares, la llama habría alcanzado una altura de 100 m y, siguiendo el recorrido del derrame, consumió alrededor de $800 \mathrm{~m}$ lineales afectando viviendas y vegetación nativa.

La causa del derrame de combustible fue una perforación realizada en la tubería como consecuencia de un robo. Los ladrones que no eran improvisados - pues contaban con tanqueros, brocas de perforación de aproximadamente una pulgada y media y mangueras especiales - abandonaron el lugar al ser descubiertos por la población que se despertó por los inusuales ruidos a esas horas. 


\section{CONSECUENCIAS DEL EVENTO}

Las consecuencias pueden ser percibidas en varios niveles de escalas (local y de la ciudad) y en diferentes órdenes: social, político, económico o ambiental6.

\section{1. Consecuencias a escala local}

Se han registrado las siguientes consecuencias como las más significativas:

\section{1. 1. En el orden social}

Este accidente pudo ser más grave dadas las condiciones del incendio, pues se estima que la radiación térmica con tendencias bajas habría alcanzado los 100 metros a la redonda. Afortunadamente, la baja densidad de viviendas alrededor de la quebrada redujo las pérdidas a nivel humano y material. El accidente dejó 2 muertos, 16 heridos y cientos de personas afectadas a las vías respiratorias y digestivas. Se evacuaron a un promedio de 300 personas; entre ellas habían 20 damnificadas que recibieron albergue. En cuanto a los daños materiales, se estima que 28 casas fueron afectadas y, de éstas, 3 fueron completamente destruidas. Otros daños se registraron en el puente que une los barrios El Girón y El Tránsito y en las vías de transporte, lo que afectó la movilidad en la zona (ver fig. 2).

\section{1. 2. En el orden económico}

Se estimaron pérdidas de alrededor de 3 millones de dólares por el combustible derramado. No existe un valor del costo por los daños de viviendas, el puente y el deterioro de las vías. Otras consecuencias de tipo económico las sufrieron 5 familias que perdieron su empleo, así como otros perjuicios en negocios y comercios de la zona a raíz de la percepción de inseguridad adquirida por este accidente. Por otra parte, también bajó el costo de la vivienda y de los terrenos?.

\section{1. 3. En el orden ambiental}

Graves daños sufrió la vegetación nativa de los bordes y taludes de un segmento importante de la quebrada El Tránsito — que había sido forestada y mantenida por el proyecto ecológico «Rescate de Quebradas» implementado por la comunidad y la Administración Zonal Quitumbe del DMQ_. Igualmente, el combustible

6 Según informes oficiales del Cuerpo de Bomberos de Quito y de PETROCOMERCIAL, recortes de prensa de El Comercio, El Universo, Ultimas Noticias, El Hoy, El Telégrafo (abril de 2003).

7 Según conversaciones mantenidas con dirigentes barriales de los barrios El Girón, El Tránsito y El Mirador (enero de 2009). 


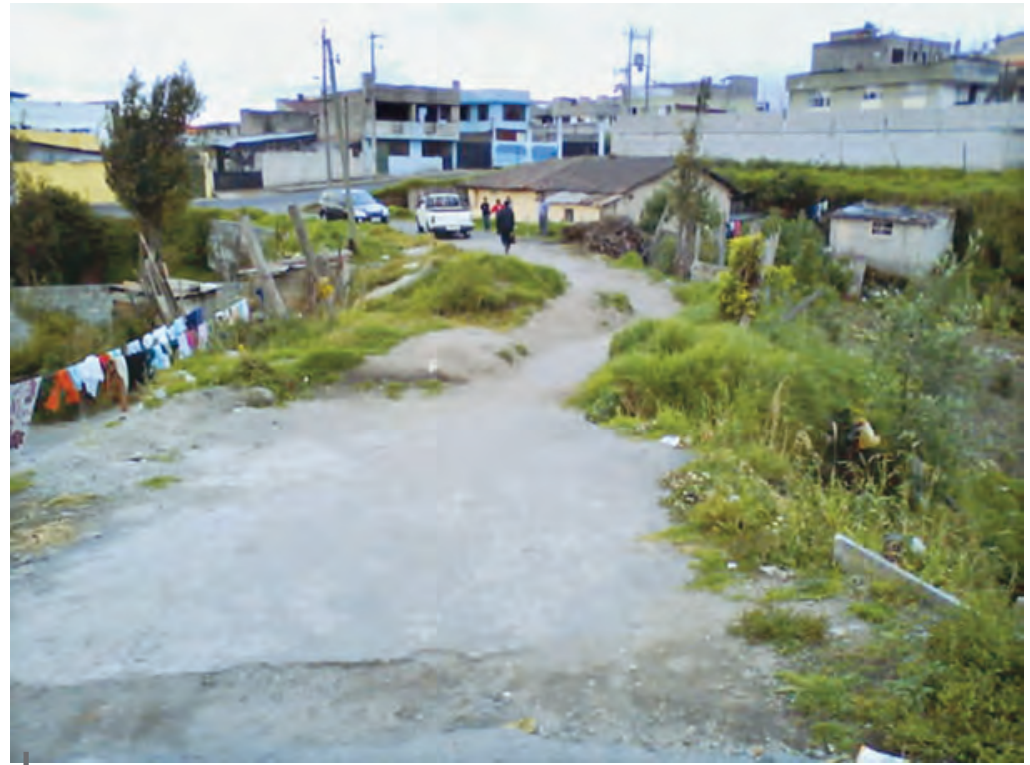

Figura 2 - Puente destruido que une los barrios El Girón y El Transito. Hasta el año 2009 aún no ha sido restaurado y solo se conserva una parte de este que es utilizado como paso peatonal

Fuente: Jairo Estacio, enero de 2009

y el incendio contaminaron el agua, lo que acarreó la imposibildiad de gestar microempresas turísticas.

\section{1. 4. En el orden político}

Se generó un conflicto institucional porque el municipio de Quito exigió a PETROECUADOR asumir el costo de las indemnizaciones pedidas por los afectados y damnificados.

\section{2. Consecuencias a escala de la ciudad}

\section{2. 1. En el orden ambiental}

Se ha comprobado una contaminación de gasolina aguas abajo de la quebrada El Tránsito hasta su confluencia con el río Machángara. Esto produjo malos olores que fueron percibidos hasta $300 \mathrm{~m}$ desde el cauce del río. Los barrios afectados fueron San Gregorio, Santa Marta de Chillogallo, Santa Inés y la parte baja de La Gatazo, Quito Sur y Teniente Ortiz. 


\section{2. 2. En el orden económico}

El poliducto roto ocasionó la interrupción del transporte de gasolina hacia El Beaterio8 por alrededor de 5 horas, ocasionando pérdidas económicas y reajustes en la distribución regional.

\section{FACTORES DE VULNERABILIDAD}

Para determinar los factores de vulnerabilidad es necesario partir de la pregunta: ¿cuáles fueron las causas que dieron origen a este desastre? Existen explicaciones, principalmente, sociales y político institucionales.

\section{1. Factores sociales}

\section{1. 1. En el orden perceptivo}

Las autoridades locales ignoran el potencial peligro generado por el transporte de combustibles, particularmente, en asentamientos humanos y zonas urbanas. Este desconocimiento no permite el control y la prevención de accidentes como derrames, fugas, incendios y explosiones, ni la evaluación de sus consecuencias para el medio ambiente y la ciudad (Leroy \& Malerba, 2005).

Además, el poliducto está enterrado casi en su totalidad, lo que lo convierte en un peligro «invisible» y poco conocido para la población. Ello desvaloriza el peligro real y es una causa por la cual la población prioriza otros problemas (la falta de servicios o la inseguridad social).

\section{1. 2. En el orden socioeconómico}

Se destacan las condiciones precarias del hábitat y la marginalidad de los barrios. Los barrios afectados se caracterizan por la presencia de viviendas precarias construidas al filo de la quebrada. Su población tiene dificultad para enfrentar situaciones generadas por los incendios debido a la falta de escaleras de salida en caso de emergencia y de hidrantes.

Por otra parte, en estos barrios de bajos niveles socioeconómicos, la falta de atención por parte de las autoridades es evidente (carencia de servicios básicos y de vías adecuadas). La condición de marginalidad hace que estos barrios sean poco visibles y gestionados en materia de desarrollo y más aún, en tema de prevención de riesgos por parte del gobierno local.

8 El Beaterio es una estación de almacenamiento de productos limpios (gasolina y diesel principalmente) que abastece no solo al DMQ sino a la región sur del país, de ahí su importancia económica y estratégica para el país. Ver el artículo de J. Estacio «Construcción y transformación del riesgo tecnológico: la terminal de combustibles El Beaterio-Quito», pp. 683-707. 


\section{2. Factores político institucionales}

\section{2. 1. En el orden de la gestión territorial}

El accidente ha revelado la falta de conocimiento sobre los peligros generados por los poliductos y de sus consecuencias e implicancias territoriales. Por ejemplo, el peligro potencial definido por parte de las autoridades petroleras y municipales llevó a la determinación de un margen de 15 metros de retiro a cada lado desde el eje principal9. Sin embargo, la experiencia del accidente demuestra que esa regulación es insuficiente, pues en ciertas condiciones meteorológicas un incendio puede generar daños en 100 metros a la redonda.

Otro factor de vulnerabilidad es la ausencia de planificación preventiva en cuanto a la ubicación de elementos de transporte de combustible, a pesar de que el Municipio del DMQ, a través de la Dirección de Planificación Territorial, ha desarrollado algunas herramientas de ocupación y uso de suelo - considerando ciertos tipos de peligro asociados a actividades industriales donde se identifican problemas de impacto ambiental_- Los ductos de transporte de hidrocarburos aún no han sido detalladamente reconocidos como una fuente de peligro para los asentamientos humanos cercanos y hacen falta mecanismos de control sobre su localización en zonas urbanas. Además, estas dinámicas tendrían que considerar no solo la población circundante sino otros aspectos físicos del territorio como las quebradas, los tipos de suelo o vegetación y otros factores relacionados con amenazas de origen natural (por ejemplo, muchos ductos han sido afectados por deslizamientos o derrumbes).

Son necesarios también los mecanismos de control para los asentamientos ilegales en zonas indebidas. Si bien existen ordenanzas que estipulan los retiros a partir de los ductos y sitios donde no se debe construir viviendas, las reglamentaciones y acciones de control son insuficientes. Es todavía muy común encontrar barrios y asentamientos en filos de quebradas o zonas de retiro y protección de poliductos (derecho de via).

\section{2. 2. En el orden político jurídico}

El incendio de Chillogallo ha evidenciado la carencia de políticas de seguridad de los poliductos y de su control externo. Este aspecto ha sido poco manejado por las autoridades petroleras en el proceso de funcionamiento de los poliductos. Una buena política de control pudo haber sido el uso de herramientas de vigilancia e intervención a distancia como una solución para reducir o evitar accidentes y pérdidas cuantiosas por robo de combustibles10, acaso un sistema de detección de fugas (Paz \& Miño, 2000).

9 Este reglamento se encuentra estipulado en el «Plan de Ocupación y Uso del Suelo (PUOS)» a cargo de la Dirección de Planificación Territorial del Municipio del Distrito Metropolitano de Quito (2008).

10 De acuerdo a dos auditorías, realizadas en PETROECUADOR por las compañías Meric Holdings Limited y Siemens en 1995 y 1999, se estima que anualmente pierde 90 millones de dólares por robo. Esta cifra seguramente aumentó a partir del año 2000 con el incremento de las perforaciones clandestinas registradas en las estadísticas de PETROCOMERCIAL. 
Finalmente, no existen políticas de desarrollo comunitario considerando los riesgos: los proyectos de reactivación social y ayuda al desarrollo local en la zona —recuperación de quebradas, de inclusión social y microempresas comunitarias— nunca han contemplado la prevención de riesgos urbanos y menos aún, los tecnológicos.

\section{DEBILIDADES Y CAPACIDADES EN LA GESTIÓN DE CRISIS}

El ente coordinador de la gestión de emergencia fue el 91111 que, a través de una llamada registrada alrededor de las 3 am del 17 de abril, convocó a los diferentes actores operativos (Cuerpo de Bomberos, Cruz Roja y Policía Nacional). El manejo de emergencia fue gestionado en cuatro etapas:

- el rescate de víctimas, la evacuación de personas y el aislamiento de la zona para evitar nuevos accidentes;

- el control del incendio a través de la extinción de la llama y el cierre de válvulas de los ductos;

- la atención a los heridos en casas asistenciales de salud;

- la evaluación del daño y la implementación de medidas posdesastre (que se detallaran más adelante).

No obstante, un evento de esta magnitud forjó aspectos positivos y negativos en la operación del manejo de emergencias y de la gestión de crisis dejando en evidencia capacidades adquiridas y también debilidades. Algunos aspectos son mencionados en el cuadro 1 de acuerdo a las etapas emprendidas. De ese cuadro sintético se desprenden algunas acotaciones importantes.

\section{1. Importancia de la hora del siniestro y de las características del incendio}

Un punto relevante del accidente es, sin duda, su ocurrencia en la madrugada. A esto se suma que, por primera vez en la ciudad, un incendio de esta magnitud tiene lugar en una quebrada colindante con viviendas y tiene un carácter expansivo por sus condiciones lineales de recorrido. Estas circunstancias pueden tener efectos positivos y negativos. Un efecto positivo destacable es que, a pesar de la lejanía de los centros de intervención y la dificultad de acceso'12, la movilización al lugar se realizó de manera rápida, debido a la inexistencia de tráfico vehicular. En cambio, las condiciones de visibilidad eran escasas en ciertos lugares, lo que limitaba las posibilidades de operar y evacuar a la población, además de su poca capacidad

11 El 911 es un organismo de atención de emergencias que coordina, además, acciones de atención con otros organismos de socorro como la Cruz Roja, el Cuerpo de Bomberos y la Policía Nacional.

12 La quebrada y la pésima calidad de las vías de comunicación no facilitaron la gestión de la crisis. La presencia de caminos, estrechos y a veces construidos en el mismo sentido que la pendiente dificultaron la labor de los carros de bomberos. 
Cuadro 1 - Capacidades y debilidades en la gestión de crisis ligada al incendio del 17 de abril del 2003 en Chillogallo

\begin{tabular}{|c|c|c|c|}
\hline $\begin{array}{l}\text { Etapas de } \\
\text { manejo de } \\
\text { crisis } \\
\end{array}$ & $\begin{array}{c}\text { Capacidades generales } \\
\text { adquiridas }\end{array}$ & Grandes debilidades detectadas & Responsables \\
\hline $\begin{array}{l}\text { Acciones } \\
\text { de rescate, } \\
\text { evacuación y } \\
\text { aislamiento } \\
\text { de personas }\end{array}$ & $\begin{array}{l}\text { La respuesta de los organismos } \\
\text { de socorro fue casi inmediata } \\
\text { a pesar de la lejanía del sector. } \\
\text { El rescate fue llevado con } \\
\text { prontitud por parte de } 10 \\
\text { ambulancias en el sector. } \\
\text { Se desplazaron a las } 4 \text { de } \\
\text { la mañana alrededor de } 16 \\
\text { heridos. } \\
\text { Defensa Civil atendió con } \\
\text { prontitud la entrega de } \\
\text { vituallas a los evacuados. }\end{array}$ & $\begin{array}{l}\text { El aislamiento del sector se realizó de } \\
\text { forma contraproducente a través de una } \\
\text { cadena humana de policías expuestos a } \\
\text { la volatilidad del incendio (por suerte no } \\
\text { hubo víctimas). } \\
\text { Los evacuados fueron llevados a } \\
\text { albergues improvisados: primero en } \\
\text { la iglesia del lugar, luego al coliseo } \\
\text { del barrio y finalmente a una escuela } \\
\text { del sector (Julio Tobar Donoso). Esto } \\
\text { evidenció la carencia de albergues } \\
\text { oficiales. } \\
\text { Faltó atención psicológica a los } \\
\text { evacuados } \\
\text { Faltaron vías de evacuación }\end{array}$ & $\begin{array}{l}\text { Cruz Roja } \\
\text { Policía } \\
\text { Nacional } \\
\text { Bomberos } \\
\text { Defensa Civil }\end{array}$ \\
\hline $\begin{array}{l}\text { Control del } \\
\text { siniestro }\end{array}$ & $\begin{array}{l}\text { El } 911 \text { coordinó } \\
\text { adecuadamente los } \\
\text { organismos de respuesta. } \\
\text { A pesar de las grandes } \\
\text { dimensiones del fuego, } \\
\text { este fue controlado con un } \\
\text { importante contingente de } \\
\text { bomberos que impidió que el } \\
\text { incendio cobrara más víctimas. }\end{array}$ & $\begin{array}{l}\text { El manejo de esta emergencia movilizó, } \\
\text { durante } 5 \text { horas aproximadamente, a } \\
\text { alrededor de } 10 \text { compañías del Cuerpo } \\
\text { de Bomberos de Quito ( } 80 \text { hombres } \\
\text { aproximadamente), así como camiones } \\
\text { cisterna del aeropuerto, personal de la } \\
\text { Cruz Roja Ecuatoriana, Policía Nacional } \\
\text { y ambulancias del } 911 \text {. Por lo tanto, si } \\
\text { se hubiera producido otro accidente en } \\
\text { el DMQ no se contaba con la suficiente } \\
\text { capacidad operativa para su atención. } \\
\text { La carencia de hidrantes a lo largo de las } \\
\text { tuberías del poliducto y en barrios causó } \\
\text { problemas de control del fuego. } \\
\text { Las válvulas de los ductos por no ser } \\
\text { automáticas, fueron cerradas } 3 \text { horas } \\
\text { más tarde del flagelo. } \\
\text { La presencia de autoridades como } \\
\text { el Alcalde de Quito y el ministro de } \\
\text { Energía y Minas ejerció una falsa } \\
\text { expectativa de oportunidades de obras } \\
\text { y servicios para la población. }\end{array}$ & $\begin{array}{l}\text { Bomberos (10 } \\
\text { compañías de } \\
\text { todo el DMQ). } \\
\text { Apoyo de la } \\
\text { DAC } \\
\text { (Dirección de } \\
\text { Aviación Civil). }\end{array}$ \\
\hline $\begin{array}{l}\text { Atención de } \\
\text { heridos en } \\
\text { centros de } \\
\text { salud }\end{array}$ & $\begin{array}{l}\text { La atención prehospitalaria fue } \\
\text { oportuna. } \\
\text { Las indemnizaciones a las } \\
\text { personas afectadas cubrieron } \\
\text { los gastos médicos. }\end{array}$ & $\begin{array}{l}\text { No se dispone de suficientes unidades } \\
\text { para quemados en los hospitales. } \\
\text { El Hospital Enrique Garcés que es el de } \\
\text { mayor demanda no dispone de unidad } \\
\text { para quemados, lo que dificultó las } \\
\text { tareas médicas. }\end{array}$ & $\begin{array}{l}\text { Hospital Baca } \\
\text { Ortiz } \\
\text { Hospital } \\
\text { Eugenio Espejo } \\
\text { Hospital } \\
\text { Enrique Garcés }\end{array}$ \\
\hline $\begin{array}{l}\text { Evaluación } \\
\text { de daños }\end{array}$ & $\begin{array}{l}\text { La aseguradora de } \\
\text { PETROECUADOR ha } \\
\text { evaluado los daños y se } \\
\text { cubren indemnizaciones hasta } \\
\text { terceros (esto contempla la } \\
\text { población afectada) }\end{array}$ & $\begin{array}{l}\text { No se realizó evaluaciones de daños } \\
\text { ambientales por considerarlos } \\
\text { menores, tampoco evaluaciones en la } \\
\text { infraestructura vial. }\end{array}$ & $\begin{array}{l}\text { PETRO- } \\
\text { ECUADOR }\end{array}$ \\
\hline
\end{tabular}

Fuentes: Informe de Incendio en el Sector de Chillogallo, Cuerpo de Bomberos de Quito, 2003. Informe de rotura en los poliductos. Unidad de Investigación y Desarrollo Tecnológico-PETROECUADOR, 2004. Investigación en diarios El Hoy, El Comercio y La Hora, abril de 2003. Elaboración: Jairo Estacio 
de reacción. Este punto resalta la importancia del factor tiempo en el manejo de crisis: de día las consecuencias hubieran sido diferentes (menor población, mejor capacidad para reaccionar, pero más tráfico para llegar a la zona) a la noche (mayor población con menor capacidad para reaccionar, pero menos tráfico).

\section{2. Una planificación de emergencia muy débil}

Existieron problemas en la planificación y atención de la emergencia debido a que, generalmente, no se consideran previsibles y prioritarios en la ciudad eventos tecnológicos como incendios, explosiones o derrames. Los barrios localizados en las cercanías del poliducto nunca han realizado simulacros ni recibido capacitación y no existen planes de evacuación masiva y de contingencia. Fue flagrante, además, la carencia de hidrantes, de vías de escape, albergues planificados (temporales y permanentes), comités poblacionales de emergencia y alarmas comunitarias contra incendios, entre otros elementos de gestión de crisis.

\section{3. El desconocimiento de factores que incrementan el peligro}

Se trata más particularmente de factores meteorológicos y poblacionales. Un incendio es una amenaza que, dependiendo de las características del material inflamable y de las condiciones meteorológicas, puede durar varias horas y hasta días (caso de incendios forestales). En este caso la cantidad de combustible retenido en la quebrada y el viento jugaron un papel importante en la intensidad como en la propagación del incendio. Se estima, según observaciones, que el fuego se elevó hasta alcanzar los 100 m y se propagó, por alrededor de 3 a 4 horas, hasta una veintena de metros hacia el sur donde se encontraba la población. En otras condiciones meteorológicas, las características y efectos de este incendio pudieron haber sido diferentes. Si el fuego se hubiera dirigido hacia el norte donde existe un mayor número de viviendas, el número de víctimas habría aumentado considerablemente.

\section{4. La escasa consideración de las secuelas psicológicas individuales y colectivas}

Según Dubois-Maury \& Chaline (2002), de todos los desastres, los ocasionados por incendios y explosiones son los que generan mayor miedo en la población. Este hecho, además de las pérdidas humanas y materiales, demuestra que, en un accidente de tales magnitudes, pueden existir trastornos y traumas individuales y colectivos que deben ser tratados dentro de la atención médica posterior al desastre. Los cuidados médicos después de este incidente no incluyeron dichas acciones. 


\section{INCIDENCIAS DEL EVENTO EN MATERIA DE PREVENCIÓN}

Generalmente se evalúan las consecuencias de los desastres en términos de elementos negativos y pérdidas. No obstante, muchas veces reflejan oportunidades de cambio positivo a mediano y largo plazo para revertir daños y errores $y$, con ello, reducir vulnerabilidades y mejorar las condiciones del desarrollo (Lavell, 2000). Existen pues diferentes tipos de incidencias reflejadas en acontecimientos a corto, mediano y largo plazo generados por políticas y acciones institucionales y poblacionales (en la figura 3 se describen algunas de ellas).

Dicha figura resalta algunos aspectos importantes que son necesarios precisar y que además generaron debates en diferentes instituciones y medios escritos como El Comercio y El Hoy.

\section{1. Control y reducción de los factores que generan la amenaza tecnológica}

Un primer aspecto se enfoca en el esfuerzo de las autoridades por investigar sobre los actores responsables de los robos. En este contexto, la pregunta que ha sido mediatizada por parte de las autoridades desde el año 2004 es iquién está detrás del robo de combustibles? El robo por «pinchazos» va más allá de los poliductos y está vinculado también con el hurto y contrabando de cilindros de gas y gasolina de camiones cisterna que son vendidos en forma clandestina en Perú y Colombia o en estaciones de servicio y de acopio ilegales del país13. Varias investigaciones emprendidas por PETROECUADOR señalan que el robo sería efectuado por personas externas en complicidad con miembros de su misma empresa.

Otro aspecto involucra las políticas de seguridad, enfocadas mayormente en la vigilancia y resguardo del poliducto. Las estadísticas de rupturas de poliductos en el periodo 2003-2008 (manejadas por PETROCOMERCIAL) revelan una disminución del $45 \%$ de rupturas por causa de robo, debido al incremento de vigilancia por parte de la Policía Nacional, del Cuerpo de Ingenieros del Ejército y de la comunidad 14 . No obstante, aún existen problemas con los derrames en los poliductos por su antigüedad u otras causas relacionadas con las amenazas de origen natural (en particular, los deslizamientos). Frente a este hecho, en 2008, PETROCOMERCIAL y ARB ECUADOR Compañía Limitada firmaron un contrato para el cambio de tubería y válvulas del Poliducto Santo Domingo-Beaterio debido a la corrosión — situación que durante 30 años ha ocasionado pérdidas económicas por fuga de combustible-.

13 Se calcula que, en 2003, existían gasolineras en la costa ecuatoriana donde el galón de gasolina súper costaba a 0,62 dólares, mientras PETROCOMERCIAL lo vende a 0,82 dólares (Diario El Hoy, marzo 2003).

14 El barrio Guamaní, localizado al sur de la ciudad, emprendió tareas comunitarias de vigilancia en ciertos tramos por donde pasa el poliducto Quito-Ambato. 
El incendio del 17 de abril de 2003 en Chillogallo (Quito)

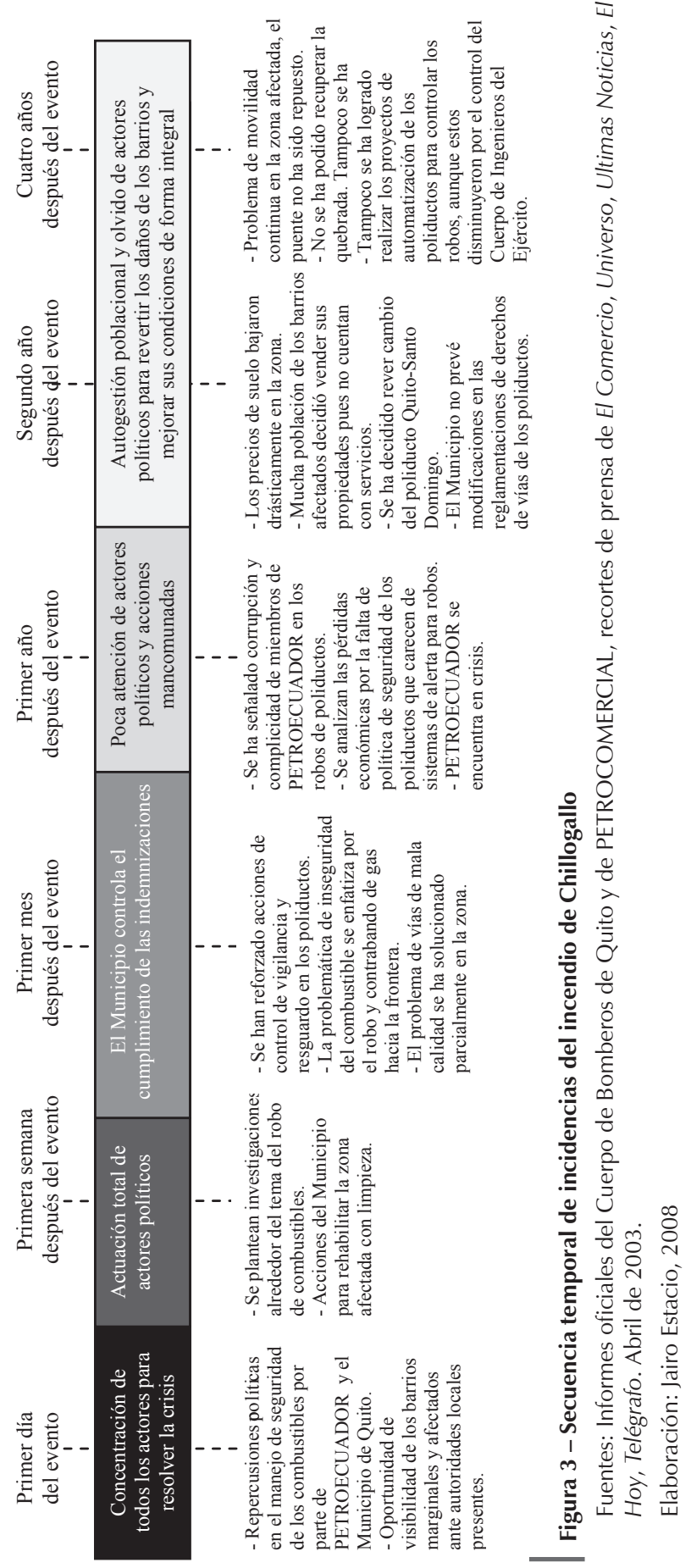


A inicios del año 2009 se inicia el «Proyecto de sistematización y automatización de los poliductos en la región Norte y Sur», manejado por PETROCOMERCIAL, que tiene como objetivo incrementar la seguridad y el control de los poliductos y terminales mediante la automatización de los sistemas de seguridad, transporte, almacenamiento, despacho y sistemas de control integral a nivel nacional15. La automatización, entre otros beneficios, permitirá detectar las fugas de combustible de manera inmediata.

\section{2. Políticas de planificación preventiva}

En esta parte se denota la ausencia de una política de planificación preventiva y de seguridad sobre el riesgo tecnológico que puede ocasionar el transporte de combustibles en el DMQ — este sería un proceso mancomunado entre el municipio, instituciones petroleras y la comunidad-. Queda demostrado que la prevención de los riesgos no siempre está asociada con los proyectos de desarrollo de la actividad hidrocarburífera o de la ciudad (Hiegel, 2003). Ello se hace evidente al observar el sector de Chillogallo donde el poliducto mantiene una seguridad mínima. En ciertos sectores, las viviendas se han incrementado en zonas aledañas al peligro y el puente destruido en la última explosión no ha sido rehabilitado.

A la vez, este incidente abre la perspectiva de considerar políticas de prevención basadas en estudios técnicos precisos. La presencia de poliductos en las ciudades significa riesgos potenciales elevados (PETROECUADOR, 2001). Por ello, se requieren normas con fundamento científico técnico para la formulación del derecho de vías, restricciones en las zonas de influencia en este tipo de equipamiento, así como planes de manejo de emergencia que preven entre otros aspectos: la construcción de vías de evacuación e hidrantes y el reforzamiento de organismos operativos para grandes eventos. Estas acciones deberían ser sostenidas con capacitaciones a la población y manejo interinstitucional, tareas que no han sido abordadas en este sector ni en otros similares.

\section{3. Reducción de la vulnerabilidad poblacional}

Surge la necesidad de políticas de reducción de la vulnerabilidad de estos barrios por sus condiciones de marginalidad, lejanía y mal acceso. Si bien las políticas de gestión urbana del DMQ consideran los riesgos y preven las formas de ocupación de suelo en zonas adecuadas - caso del Plan de Ocupación y Uso de Suelos (PUOS) del año 2008-, se deben iniciar planes de gestión específicos para el desarrollo local en materia de servicios, movilidad, seguridad y desarrollo social. Sin embargo, no se ha generado aún una política de desarrollo barrial que apunte no solo a las zonas de mayor visibilidad política, económica o patrimonial, sino también a las zonas más vulnerables desde el punto de vista social y territorial donde los desastres son mayores y frecuentes - ello podría contener cierta discriminación social y territorial hacia ciertos barrios-. 


\section{4. De la crisis a la oportunidad de cambio}

De manera general, se esperaba que la trascendencia del incendio, en términos de ocurrencia y afectación (uno de los más graves ocurridos en la capital), abriera el debate sobre la incorporación de los riesgos tecnológicos en las agendas de planificación y desarrollo. Así se podría concentrar una visión más integral de la reducción de los riesgos urbanos en el DMQ. No obstante, el balance de lo ocurrido, de manera limitada, se ha concentrado en acciones vinculadas al campo reactivo del riesgo (control de la amenaza, vigilancia de poliductos, entre otros) y no al campo correctivo en términos de reducción de vulnerabilidades (como evitar la presencia de asentamientos ilegales en zonas de riesgo tecnológico, que además carecen de servicios básicos, o educar tanto a la población como a las autoridades sobre las implicancias del peligro tecnológico).

En un inicio, la presencia de desastres normalmente hace visibles las necesidades de un sector, lo que debería permitir cambios positivos en las zonas que anteriormente no eran o eran poco gestionadas territorialmente por parte de las autoridades. Sin embargo, eso no se realizó del todo en los barrios afectados. Si bien el accidente convocó la presencia de autoridades en la zona16, quienes en primera instancia se comprometieron en mejorar las condiciones del barrio —en circunstancias normales no habría sucedido-, las promesas no se cumplieron y las acciones fueron mínimas. En la actualidad existen todavía vulnerabilidades sociales, ambientales, económicas y físicas que no han podido ser revertidas.

Al comparar los barrios afectados de Chillogallo con otros similares - como los de las laderas noroccidentales del Pichincha de Quito, en donde se han generado desastres naturales por deslizamientos y derrumbes principalmente-, se evidencia que éstos últimos han encontrado situaciones de cambio real y desarrollo después de los desastres. Dos de estos barrios son Atucucho y Nueva Aurora, donde se construyeron obras para la mitigación y mejoras en equipamientos y movilidad.

Tomando en cuenta la particularidad del mejoramiento barrial luego de un desastre, cabe la pregunta ipor qué en los barrios afectados de Chillogallo no se han sostenido acciones de cambio y de reducción de vulnerabilidades? Si bien pueden existir varias causas relacionadas a factores sociales, políticos y económicos, existen algunas que sobresalen como las siguientes:

- La falta de continuidad en las acciones de gestión barrial por parte de la población, debido al debilitamiento de la organización barrial, sumada a la venta y arrendamiento de varias propiedades después del accidente. Ello detuvo el interés de continuar de forma más cohesionada las labores de autogestión local.

- El bajo nivel de ocurrencia de estos accidentes fragilizan la memoria colectiva. Al comparar los accidentes tecnológicos con los deslizamientos o inundaciones que son más frecuentes, los primeros son muy eventuales y aleatorios. Por lo tanto, disminuye la atención en la zona que fue afectada.

16 Luego del accidente, el lugar fue visitado por el alcalde del DMQ, Gral. Paco Moncayo, el ministro de Energía y Minas, Carlos Arboleda, y el presidente de PETROCUADOR. 
- El poco interés que representa la zona afectada puede ser una causa que impide priorizar acciones para su mejoramiento. Acaso tiene una vocación eminentemente residencial con pocos elementos urbanos que coadyuven el desarrollo a escala de la ciudad (salvo el mismo poliducto que representa un elemento de interés mayor).

- El imaginario urbano y social de los barrios del sur del DMQ en el contexto del desarrollo de la ciudad. Desde que la ciudad de Quito fue sometida a un proceso de planificación, el norte fue considerado como una zona residencial y de crecimiento económico, mientras el sur era un sector marginal, popular y «obrero»17, donde se asentaban grupos poblacionales de bajos recursos económicos. Si bien, actualmente, existe la concepción de marginalidad y pobreza circunscrita a muchos barrios periféricos localizados en diferentes zonas del DMQ, las zonas afectadas por el accidente presentan esta doble concepción; es decir, son barrios marginales y localizados al sur de Quito. Aunque en la realidad deberían tener mayor prioridad, todavía carecen de obras de mitigación y de servicios y equipamientos básicos que aún no han sido gestionadas por las autoridades locales.

Con estas acotaciones se demuestra que el accidente de Chillogallo, a pesar de su intensidad y gravedad, no fue suficiente para generar un cambio de mentalidad en las formas de conocimiento e intervención de los riesgos tecnológicos en el DMQ. No obstante, deja constancia de los efectos de las fuentes de peligro relacionadas con espacios urbanos y su fuerte incertidumbre vinculada a factores políticos, sociales y territoriales para controlarlos, así como los nuevos retos de una ciudad para reducirlos en el marco del desarrollo local.

17 Esta concepción tuvo su origen en el Plan de Quito Jones Odriozola de 1942, la misma que marcó el imaginario del sur como de vocación obrera y popular. 


\section{Referencias citadas}

DUBOIS-MAURY, J. \& CHALINE, C.,2002 - Les Risques Urbains, 208 pp.; París: Éditions Armand Colin.

CUERPO DE BOMBEROS DE QUITO, 2003 - Informe de Incendio en el Sector de Chillogallo 2003, 10 pp.; Quito: MDMQ.

ESTACIO, J., 2004 - Risques technologiques liés au stockage et au transport de combustibles dans le District Métropolitain de Quito, 91 pp.; Chambéry: Université de Savoie. Mémoire de DEA.

HIEGEL, C., 2003 - Des risques urbains méconnus: Les risques technologiques mineurs, exemple des stations-service a Strasbourg, 400 pp.; Strasbourg: Université Louis Pasteur Strasbourg I, Laboratoire Image et ville, CNRS. Thèse de géographie.

LAVELL, A., 2000 - Desastres y Desarrollo: Hacia un Entendimiento de las Formas de Construcción Social de un Desastre: El Caso del Huracán Mitch en Centroamérica, 25 pp.; Lima, La Red.

LEROY, J. \& MALERBA, J., 2005 - Integración o Explotación, 130 pp.; Quito-Rio de Janeiro Brasil: Asesoría Gráfica. Projeto Brasil Sustentável e Democrático.

MDMQ, 2008 - Plan de Ocupación y Uso del Suelo, 98 pp.; Quito-Ecuador: Dirección de Planificación Territorial-MDMQ.

PAZ Y MIÑO, C., 2000 - Daños sin Reparaciones de Hidrocarburos, 2 pp.; Quito: Escuela Politécnica Nacional. Artículo publicado en cartelera de la facultad de Petróleos.

PETROECUADOR, 2001 - Normas de incidentes y accidentes de trabajo, 24 pp.; Quito Ecuador: Unidad de Seguridad e Higiene Industrial. 
